\title{
Carbon Ignition in Type Ia Supernovae: An Analytic Model
}

\author{
S. E. Woosley \\ Department of Astronomy and Astrophysics, University of California, Santa Cruz, CA 95064 \\ woosley@ucolick.org \\ S. Wunsch \\ Combustion Research Facility \\ Sandia National Laboratories, Livermore, CA 94551-0969 \\ sewunsc@ca.sandia.gov \\ and \\ M. Kuhlen \\ Department of Astronomy and Astrophysics, University of California, Santa Cruz, CA 95064 \\ mqk@ucolick.org
}

\begin{abstract}
The observable properties of a Type Ia supernova are sensitive to how the nuclear runaway ignites in a Chandrasekhar mass white dwarf - at a single point at its center, off-center, or at multiple points and times. We present a simple analytic model for the runaway based upon a combination of stellar mixing-length theory and recent advances in understanding RayleighBenard convection. The convective flow just prior to runaway is likely to have a strong dipolar component, though higher multipoles may contribute appreciably at the very high Rayleigh number $\left(10^{25}\right)$ appropriate to the white dwarf core. A likely outcome is multi-point ignition with an exponentially increasing number of ignition points during the few tenths of a second that it takes the runaway to develop. The first sparks ignite approximately $150-200 \mathrm{~km}$ off center, followed by ignition at smaller radii. Rotation may be important to break the dipole asymmetry of the ignition and give a healthy explosion.
\end{abstract}

Subject headings: Supernovae, hydrodynamics

\section{INTRODUCTION}

Despite forty years of study (Hoyle \& Fowler 1960), the mechanism whereby a degenerate carbon-oxygen white dwarf explodes, producing a Type Ia supernova (SN Ia), remains poorly understood (for a recent review see Hillebrandt \& Niemeyer 2000). Early calculations assumed that central carbon ignition would lead to a detonation (Arnett 1969) that would incinerate the star entirely to iron. This proved inconsistent both with observations of features in the supernova spectrum from intermediate mass elements and with detailed calculations of isotopic nucleosynthesis. Nowadays it is understood that prompt detonation does not occur because the core at ignition time is insufficiently isothermal (Woosley 1992).

Attention in recent years has thus focused on deflagrations, subsonic burning fronts in which pressure equilibrium is maintained across the burning interface. Though it is controversial whether the deflagration will later make a transition to a detonation (Niemeyer \& Woosley 1997; Khokhlov, Oran, \& Wheeler 1997; Niemeyer 1999), it is universally assumed that the runaway 
begins as a deflagration (Nomoto, Sugimoto, \& Neo 1976).

There is less certainty, though, regarding exactly how and where the runaway is ignited. Most one-dimensional (1D) calculations, because of their imposed symmetry, obtain ignition at the center of the star. Many 2D studies have also assumed central ignition, largely as a matter of convenience. However recent work (Niemeyer, Hillebrandt, \& Woosley 1996; Reinecke, Hillebrandt, \& Niemeyer 1999, 2002ab) has highlighted the sensitivity of the supernova outcome to precisely how the runaway is initiated - at the center or at one or more points off-center. These results may be summarized as showing that, in the absence of detonation, multi-point spherically-symmetric offcenter ignition gives the most robust explosions, central single-point ignition gives weaker ones, and single-point off-center ignition gives such a weak explosion that it fails to unbind the white dwarf on the first attempt.

It has been recognized for some time that the critical circumstances affecting the ignition are determined when the convecting white dwarf core reaches a density of $2-3 \times 10^{9} \mathrm{~g} \mathrm{~cm}^{-3}$ and a temperature of about $7 \times 10^{8} \mathrm{~K}$ (Nomoto et al 1984; Woosley \& Weaver 1986). For these conditions, the time scale for the increase of nuclear energy generation becomes comparable to the convective turnover time, both of order 10 to $100 \mathrm{~s}$. By the time any fluid element reaches $10^{9} \mathrm{~K}$, burnings has become quicker even than the time it takes a sound wave to cross a pressure scale height and, for all practical purposes, carbon burns instantly to iron. The surface of this fluid element then becomes a "flame", with a well determined speed (Timmes \& Woosley 1992) and a buoyancy given by its density decrement, $\Delta \rho / \rho \sim 15 \%$. The explosion is born.

Woosley (1990) first suggested that the much smaller buoyancy of convective fluid elements would still lead to appreciable radial motion, even as the convection decoupled from the burning. Thus ignition would occur off-center at one or more points already moving rapidly outwards. This speculation was rendered more quantitative by Garcia-Senz \& Woosley (1995), who, using a simple parameterized description of "burning floating bubbles", estimated a typical ignition radius $\sim 200 \mathrm{~km}$. They also noted that off-center ignition would help alleviate a chronic overpro- duction of neutron-rich isotopes in Type Ia supernovae, since most of the burning would take place farther out at lower density than in centrally ignited models.

Woosley (2001) estimated the convection speeds $\left(\sim 100 \mathrm{~km} \mathrm{~s}^{-1}\right)$ and temperature fluctuations $(\Delta T / T \sim 0.3-3 \%)$ in the convective core at the time of runaway and, comparing them to deflagration flame speeds and accelerations due to off-center burning, all of which are comparable, concluded that multi-point off-center ignition was probable. He also pointed out that the exact number and location of points was sensitive to small variations in the initial conditions, thus introducing some degree of chaos in the outcome. Since such observables as the kinetic energy, nickel mass and peak luminosity are sensitive to how the star ignites, Type Ia supernovae, starting from nearly identical initial conditions, will always exhibit some irreducible diversity.

The conclusion that multi-point off-center ignition is likely was challenged by Höflich \& Stein (2002). Using a 2D implicit hydrodynamics code to follow the last few hours of the white dwarf runaway, they found no evidence for multiple spot or strong off-center ignition. Instead their model ignited at about $30 \mathrm{~km}$, virtually at the center, and only once. Ignition was "induced by compressional heat". As we shall see, their results, though a major computational advance, may have been influenced by attempting to model a $3 \mathrm{D}$, spherical problem while carrying only a fraction of the solid angle on a 2D grid. The resolution and Reynolds number may also have been too low to see multipoint ignition in a simulation that was, at best, mildly turbulent and included only a small fraction of the fluctuation distribution function for the temperature.

In this paper and its companion (Kuhlen, Woosley, \& Glatzmaier 2003a; Paper II), we explore the ignition of a nuclear runaways in Chandrasekhar mass white dwarfs using two different approaches - an analytic model (this paper) and a 3D anelastic numerical model. In the analytic case, we are influenced by recent developments in understanding convection in Rayleigh-Benard experiments. These experiments show that the qualitative character of convection may change markedly depending upon the Rayleigh number. Even at high Rayleigh number, a persistent "roll" 
dominates the flow pattern in Rayleigh-Benard convection. The analogue to this in a sphere is a dipole flow pattern. Recent numerical simulations (Paper II) suggest that such a large scale flow is also present in (non-rotating) convective stars. If so, it affects the mechanics of white dwarf ignition in a major way that can only be seen by carrying the entire sphere in the calculation.

\section{AN ANALYTIC MODEL OF THE RUNAWAY}

The final stages of the carbon runaway, wherein roughly $1.1 \mathrm{M}_{\odot}$ of the core becomes convective, goes on for well over a century. By the time the central temperature reaches $T_{8}=T / 10^{8} \mathrm{~K}=7$ and $\rho_{9}=\rho / 10^{9} \mathrm{~g} \mathrm{~cm}^{-3}=2$, the typical time scale for convection to go a pressure scale height, about 450 $\mathrm{km}$, is $\sim 10 \mathrm{~s}$, and has become comparable to the nuclear time scale.

\subsection{Nuclear energy generation rate and time scale}

Nuclear energy generation during carbon ignition is given entirely by the (highly screened) fusion of two ${ }^{12} \mathrm{C}$ nuclei to form, chiefly, ${ }^{20} \mathrm{Ne}$ and ${ }^{24} \mathrm{Mg}$. The approximate energy generation rate (assuming carbon burns to a mixture of 3 parts ${ }^{20} \mathrm{Ne}$ and one part ${ }^{24} \mathrm{Mg}$; Woosley 1986) is

$$
\begin{gathered}
\dot{S}_{\text {nuc }} \approx 6.7 \times 10^{25} \mathrm{X}^{2}\left({ }^{12} \mathrm{C}\right) \rho_{9} F_{\text {sc }} \lambda_{12,12} \\
\operatorname{erg~g}^{-1} \mathrm{~s}^{-1},
\end{gathered}
$$

where $\lambda_{12,12}$ is the carbon fusion reaction rate (Caughlan \& Fowler, 1988), $F_{\text {sc }}$ is the electron screening function, and $\mathrm{X}\left({ }^{12} \mathrm{C}\right)$ is the mass fraction of carbon. For a range of temperatures, $T_{8}=$ $6-8$,

$$
\lambda_{12,12} \approx 7.6 \times 10^{-16}\left(\frac{T_{8}}{7}\right)^{30}
$$

The electron screening function (Alastuey \& Jancovici, 1978) is given by $\left(\rho_{9}=1-3 ; T_{8}=6-\right.$ 8)

$$
F_{\mathrm{sc}} \approx 1100\left(\frac{\rho_{9}}{2}\right)^{2.3}\left(\frac{T_{8}}{7}\right)^{-7}
$$

so that the energy generation rate for a composition of $50 \%$ carbon, $50 \%$ oxygen is

$\dot{S}_{\text {nuc }} \approx 2.8 \times 10^{13}\left(\frac{T_{8}}{7}\right)^{23}\left(\frac{\rho_{9}}{2}\right)^{3.3} \mathrm{erg} \mathrm{g}^{-1} \mathrm{~s}^{-1}$.

The specific energy available is

$$
q_{\text {nuc }}=4.0 \times 10^{17} \mathrm{X}\left({ }^{12} \mathrm{C}\right) \mathrm{erg} \mathrm{g}^{-1} .
$$

The specific heat at constant pressure, which is required to estimate the nuclear time scale, is (e.g., Chiu 1968)

$$
\begin{gathered}
c_{\mathrm{P}}=\left(\frac{\partial \epsilon}{\partial T}\right)_{\text {ions }}+\left(\frac{\partial \epsilon}{\partial T}\right)_{\text {electrons }}+\left(\frac{\partial \epsilon}{\partial T}\right)_{\text {radiation }} \\
=\left(\frac{3 N_{\mathrm{A}} k}{2 \bar{A}}\right)+\frac{\pi^{2} k^{2}}{x m_{e} c^{2}} \rho N_{\mathrm{A}} Y_{e} T+\left(\frac{4 a T^{3}}{\rho}\right) \\
=9.1 \times 10^{14}+\frac{8.6 \times 10^{13} T_{8}}{\rho_{9}^{1 / 3}} \\
+\frac{3.0 \times 10^{9} T_{8}^{3}}{\rho_{9}} \mathrm{erg} \mathrm{g}^{-1}\left(10^{8} \mathrm{~K}\right)^{-1},
\end{gathered}
$$

where $\bar{A}$ is the mean atomic mass number (13.7 for $50 \%$ carbon, $50 \%$ oxygen by mass); $m$ is the mass of the electron, $x=p_{\mathrm{F}} / m c$ is related to the mass density by $9.74 \times 10^{5} \mu_{e} x^{3}=\rho ; Y_{e}$ is the electron mole number, here 0.5 ; and the other symbols have their usual meanings. For the conditions of interest, e.g., $T_{8}=7$ and $\rho_{9}=2$, the heat capacity of the radiation field is negligible and the ions and electrons together provide $1.4 \times 10^{15} \mathrm{erg}$ $\mathrm{g}^{-1}\left(10^{8} \mathrm{~K}\right)^{-1}$. This relatively small heat capacity makes the carbon highly incendiary in the sense that a small amount of burning raises the temperature considerably. For lower densities, however, $\rho_{9} \sim 0.01$, the heat capacity of the radiation field becomes important, and this is what finally keeps the star from burning entirely to iron.

For central temperatures near $T_{o, 8}=7$, The 
nuclear time under these conditions is

$$
\begin{aligned}
\tau_{\text {nuc }} & =\left(\frac{1}{\dot{S}_{\mathrm{nuc}}} \frac{d \dot{S}_{\mathrm{nuc}}}{d t}\right)^{-1} \\
& \approx\left(\frac{1}{\dot{S}_{\mathrm{nuc}}} \frac{\partial \dot{S}_{\mathrm{nuc}}}{\partial T} \frac{\partial T}{\partial t}\right)^{-1} \\
& =\frac{c_{\mathrm{P}} T}{23 \dot{S}_{\mathrm{nuc}}} \\
& \approx 15\left(\frac{7}{T_{8}}\right)^{22}\left(\frac{2}{\rho_{9}}\right)^{3.3} \mathrm{~s} .
\end{aligned}
$$

This is the time for the energy generation in an isolated region to increase from its starting value at temperature, $T_{8}$, to such high values that the reactions are virtually instantaneous. While convection remains efficient, this time is lengthened in the star by a factor of approximately 50 (§ 4.1).

\subsection{Luminosity}

The long convective episode in the pre-explosive star establishes an overall adiabatic temperature gradient in the central regions. Using this condition, the known density structure, energy generation, Eq.(4), and assuming hydrostatic equilibrium, one can estimate the luminosity.

Because of the extreme sensitivity of the energy generation to temperature, the luminosity will originate from a small fraction of the mass justifying a first order polytropic extrapolation $(n$ $=3$ ) of the central conditions. The equation of state is that of a relativistically degenerate gas,

$$
\begin{aligned}
P & =K \rho^{4 / 3} \\
& =1.24 \times 10^{27}\left(\frac{\rho_{9}}{2}\right)^{4 / 3} \text { dyne } \mathrm{cm}^{-2},
\end{aligned}
$$

and the polytropic radius parameter,

$$
\begin{aligned}
a & =\left(\frac{K}{\pi G \rho_{o}^{2 / 3}}\right)^{1 / 2} \\
& =385 \mathrm{~km}\left(\frac{2}{\rho_{o, 9}}\right)^{1 / 3}
\end{aligned}
$$

with $\rho_{o, 9}$ the central density in $10^{9} \mathrm{~g} \mathrm{~cm}^{-3}$. Defining a dimensionless radius, $\zeta=r / a$, the mass interior to radius $r$ is given by $\left(\zeta^{2} \ll 1\right)$,

$$
M(r) \approx \frac{4 \pi}{3} r^{3} \rho_{o}\left(1-\frac{3}{10} \zeta^{2}\right)
$$

and the density at radius $r$ is

$$
\rho(r) \approx \rho_{o}\left(1-\frac{1}{2} \zeta^{2}\right) .
$$

Combining the equation of hydrostatic equilibrium

$$
\frac{d P}{d r}=-\frac{G M(r) \rho(r)}{r^{2}},
$$

with the condition for an adiabatic temperature gradient

$$
\frac{d T}{d r}=\left(1-1 / \Gamma_{2}\right)(T / P) \frac{d P}{d r},
$$

one obtains, for $\Gamma_{2} \approx 1.7$, the variation of temperature with radius near the center of the white dwarf (Woosley 1990)

$$
\begin{aligned}
T(r) & \approx T_{o}\left(1-\frac{\Gamma_{2}-1}{\Gamma_{2}} \frac{2 \pi G \rho_{o}^{2} r^{2} f_{1}}{3 P_{o}}\right) \\
& \approx T_{o}\left(1-0.0185\left(\frac{\rho_{o, 9}}{2}\right)^{2 / 3} f_{1} r_{7}^{2}\right),
\end{aligned}
$$

where $f_{1} \approx\left(1-\frac{1}{15} \zeta^{2}\right)$ is a correction, near unity, for the density gradient. This equation also describes the temperature evolution of any adiabatically expanding (or contracting) fluid element as it moves in a region near the star's center.

One may then integrate Eq.(14) to obtain the luminosity as a function of central temperature,

$$
\begin{aligned}
L & =4 \pi \int \dot{S}_{\text {nuc }} \rho r^{2} d r \\
& \approx 7.0 \times 10^{44} \mathrm{erg} \mathrm{s}^{-1}\left(\frac{\rho_{o, 9}}{2}\right)^{4.3}\left(\frac{T_{o, 8}}{7}\right)^{23} I,
\end{aligned}
$$

where $I$ is the integral,

$$
I=\int r_{7}^{2}\left(1-b r_{7}^{2}\right)^{23} f_{2} d r_{7},
$$

with $b=0.0185\left(\rho_{9} / 2\right)^{2 / 3} f_{1}$ and $f_{2} \approx\left(1-\frac{1}{2} \zeta^{2}\right)^{4.3}$. This integral can be evaluated numerically to give $I=0.98$ and 0.65 for $\rho_{o, 9}=2$ and 3 respectively. The result for the luminosity is valid to better than $20 \%$.

One can also estimate the size of the energy generating region by calculating the radius where $L$ reaches one-half its value, 140 and $120 \mathrm{~km}$ respectively for $\rho_{9}=2$ and 3 . That is, approximately one-half of the luminosity of the star is generated in its inner $130 \mathrm{~km}$. 


\subsection{Results from mixing-length theory}

As the runaway proceeds, the central temperature rises and, along with it, the luminosity. Energy is transported by convection and dissipated by expansion, i.e., $P d V$ work against gravity, and neutrinos, both of which occur chiefly outside the energy-generating central core.

Conditions near the end of this ramp up can be estimated using mixing-length theory (e.g., Clayton 1983; Lantz \& Fan 1999). In particular, the heat flux at radius $r$ is given by

$$
\begin{aligned}
\phi(r) & =\frac{L(r)}{4 \pi r^{2}} \\
& =\frac{\rho}{2} v_{\mathrm{rms}} c_{\mathrm{P}}(l \Delta \nabla T) \\
& \approx \frac{\rho}{2} v_{\mathrm{rms}} c_{\mathrm{P}}(\Delta T)
\end{aligned}
$$

where $L(r)$ is the luminosity at radius $r, \rho$ is the density, $v_{\text {rms }}$, the average convective velocity there, $c_{\mathrm{P}}$, the heat capacity, Eq.(6), $l$, a characteristic size for the convection region, and $\Delta T$, the temperature change across this region in excess of the adiabatic value. The factor " $\frac{1}{2}$ " accounts for the fact that heat is carried by the outward moving fluid elements, while lower entropy elements return the mass. Density is assumed nearly constant in all elements at a given radius.

Fluid elements are buoyant because, at constant pressure, their excess temperature is accompanied by a deficiency in density. The logarithmic derivative of density with respect to temperature, at constant pressure, is given by

$$
\begin{aligned}
\delta_{\mathrm{P}} & =-\left(\frac{\partial \ln \rho}{\partial \ln T}\right) \\
& =\frac{T}{\rho} \frac{\Delta \rho}{\Delta T} \\
& =\frac{T}{\rho}\left(\frac{\partial \mathrm{P}}{\partial T}\right)_{\rho}\left(\frac{\partial \mathrm{P}}{\partial \rho}\right)_{T}^{-1} \\
& \approx 1.9 \times 10^{-2} \frac{T}{\rho}
\end{aligned}
$$

for a range of temperatures near $T_{8}=7$ and $\rho_{9}=2$. The derivatives in the above equation were evaluated numerically using the equation of state in the Kepler code (Weaver, Zimmerman, \& Woosley 1978). For example, for $\rho_{9}=2$ and at $T_{8}=7$ and 8 respectively, $\delta_{\mathrm{P}}=6.6 \times 10^{-3}$ and
$7.7 \times 10^{-3}$ respectively. The small value of this dimensionless constant reflects the extreme degeneracy of the gas, i.e., that a large temperature change is required to give a pressure change comparable to that resulting from a small change in density.

A typical convective velocity is then given by

$$
v_{\mathrm{rms}} \approx\left(\frac{2 g \Delta \rho}{\rho}\right)^{1 / 2} l^{1 / 2},
$$

with $g$, the local acceleration due to gravity, i.e., $G M(r) / r^{2}$, and $\Delta \rho$, the density variation corresponding to $\Delta T$. Combining Eqs. (17), (18), and (19), one has the mixing-length-theory estimate for the typical convection speed,

$$
\begin{aligned}
v_{\mathrm{rms}} & \approx\left(\frac{4 g r \delta_{\mathrm{P}} \phi(r)}{\rho c_{\mathrm{P}} T}\right)^{1 / 3} \\
& \approx\left(\frac{4 G \delta_{\mathrm{P}} L}{3 c_{\mathrm{P}} T}\right)^{1 / 3} .
\end{aligned}
$$

Here $L$ is the luminosity in erg $\mathrm{s}^{-1}$. So long as $L$ is nearly constant, the result is insensitive to $l$.

$$
\text { For } \begin{aligned}
L_{45} & =L(200 \mathrm{~km}) / 10^{45} \mathrm{erg} \mathrm{s}^{-1}, \\
v_{\mathrm{rms}} & \approx 40 \mathrm{~km} \mathrm{~s}^{-1}\left(\frac{7}{T_{o, 8}}\right)^{1 / 3} L_{45}^{1 / 3}
\end{aligned}
$$

Since $L$ is zero at the stellar center, $v_{\text {rms }}$ formally goes to zero there, but its actual value depends upon the pattern of fluid flow. As is discussed in $\S 3.2$, the velocity just outside the energy generating core, say at $100-200 \mathrm{~km}$, may be a characteristic of the entire convection region.

Most of the temperature dependence of $v_{\mathrm{rms}}$ is contained in the $L / T$ term. This depends on the central conditions roughly as $T_{o}^{22} \rho_{o}^{4.3}$, so the velocity scales with the central temperature roughly as $T_{o}^{7} \rho_{o}^{1.4}$. From Eq.(15) the luminosity for $T_{o, 8}=7, \rho_{9}=2$ is about $10^{45} \mathrm{erg} \mathrm{s}^{-1}$, so

$$
v_{\mathrm{rms}} \approx 40 \mathrm{~km} \mathrm{~s}^{-1}\left(\frac{\rho_{9}}{2}\right)^{1.4}\left(\frac{T_{o, 8}}{7}\right)^{7} .
$$

That is, one expects speeds about 2.5 times faster, or $100 \mathrm{~km} \mathrm{~s}^{-1}$, at $T_{o, 8}=8$ compared with $T_{o, 8}=7$.

The corresponding characteristic temperature excess across the convection zone is

$$
\Delta T=\left(\frac{2 \phi^{2} T}{\rho^{2} c_{\mathrm{P}}^{2} g l \delta_{\mathrm{P}}}\right)^{1 / 3},
$$


or equivalently,

$$
\begin{aligned}
\frac{\Delta T}{T} & =\frac{v_{\mathrm{rms}}^{2}}{2 g l \delta_{\mathrm{P}}} \\
& =0.005\left(\frac{\rho_{9}}{2}\right)^{2.1}\left(\frac{T_{o, 8}}{7}\right)^{14} .
\end{aligned}
$$

This is the change in temperature excess across the region, beyond the adiabatic value. It is much smaller than the total change in temperature across the convection zone.

The typical density variation, $\Delta \rho / \rho$, is $\delta_{\mathrm{P}}$ times this, or about $3.6 \times 10^{-5}$. Because of the uncertain choice of $l$ and the use of a single energy characterized by $\Delta T$ to carry the flow, these estimates are probably only accurate to a factor of two. There may also be some weak dependence of $\Delta T$ on the Rayleigh number not included in our simple analysis.

As we shall see later, it is a characteristic of the higher temperature fluctuations that ignite the explosion that they move with a higher speed than the average, $v_{\text {rms }}$. Also at $T_{o, 8}=7.5-8.0$ which may be a more typical choice for the mean central temperature at ignition, $v_{\mathrm{rms}} \sim 100 \mathrm{~km} \mathrm{~s}^{-1}$. It is interesting, and seemingly an accident of nature, that, to about a factor of two, this is the same as the conductive laminar flame speed (Timmes \& Woosley 1992),

$$
u_{\mathrm{lam}} \approx 76 \mathrm{~km} \mathrm{~s}^{-1}\left(\frac{\rho_{9}}{2}\right)^{0.805}\left(\frac{\mathrm{X}\left({ }^{12} \mathrm{C}\right)}{0.5}\right)^{0.889} \text {. }
$$

Partly because of this coincidence there is a dependence of the outcome on the carbon mass fraction in the white dwarf interior.

\subsection{Some characteristic measures}

The opacity, given by electron conduction (Timmes 2000 and 2002, private communication), is

$$
\kappa_{\mathrm{cond}} \approx 2.7 \times 10^{-5} \mathrm{~cm}^{2} \mathrm{~g}^{-1}\left(\frac{2}{\rho_{9}}\right)^{1.4}\left(\frac{T_{8}}{7}\right)^{2.2} \text {. }
$$

Using this to get the conductivity,

$$
\sigma=\frac{4 a c T^{3}}{\rho \kappa}
$$

for typical conditions, $\sigma \approx 3 \times 10^{18} \mathrm{erg} \mathrm{cm}^{-1} \mathrm{~K}^{-1}$ $\mathrm{s}^{-1}$. The viscosity, $\eta$, is given (Nandkumar \&
Pethick 1984) by

$$
\eta \approx \frac{1.9 \times 10^{9}}{Z}\left(\frac{\rho_{9}}{2}\right) I_{2}^{-1} \mathrm{~g} \mathrm{~cm}^{-1} \mathrm{~s}^{-1},
$$

where $I_{2} \approx 0.5$ and $Z \approx 7$. Hence $\eta \approx 10^{9} \mathrm{~g} \mathrm{~cm}^{-1}$ $\mathrm{s}^{-1}$. From these one can calculate a Rayleigh number (the so called "dimensionless temperature gradient" in convection; the ratio of buoyancy forces to diffusion forces)

$$
\begin{aligned}
R a & =\frac{g l^{3} \rho^{2} c_{\mathrm{P}} \delta_{\mathrm{P}} \Delta T}{T \eta \sigma} \\
& \sim 10^{25}
\end{aligned}
$$

where we have assumed that the appropriate $\Delta T$ is approximately $(\Delta \nabla T) l$, Eq. (17), from mixing length theory. One can also estimate a Prandtl number (ratio of momentum transport to heat conduction)

$$
\begin{aligned}
\operatorname{Pr} & =\frac{c_{\mathrm{P}} \eta}{\sigma} \\
& \sim 4 \times 10^{-3},
\end{aligned}
$$

a Reynolds number (ratio of inertial forces to viscous forces),

$$
\begin{aligned}
R e & =\frac{\rho v_{\mathrm{rms}} l}{\eta} \\
& \approx 10^{14}
\end{aligned}
$$

and a Kolmogorov length (where turbulence dissipates),

$$
\begin{aligned}
L_{\mathrm{Kol}} & =l R e^{-3 / 4} \\
& \approx 3 \times 10^{-4} \mathrm{~cm} .
\end{aligned}
$$

This combination of Rayleigh and Prandtl numbers is well beyond the limits of what can be studied on the Earth by either experiment or simulation.

One can also estimate the Nusselt number (total rate of heat transfer compared with conduction),

$$
\begin{aligned}
N u & =\frac{\phi l}{\sigma \Delta T} \\
& \approx \frac{\rho v_{\mathrm{rms}} l c_{\mathrm{P}}}{2 \sigma} \\
& \approx 3 \times 10^{11}
\end{aligned}
$$

which will be relevant in later discussions. 


\section{LESSONS FROM RAYLEIGH-BENARD CONVECTION}

\subsection{Recent Developments}

There exists a rich literature of experiments and simulations that study the convection of matter between a hot and a cold plate. An important issue is how the relation between heat transport and the Rayleigh number, called $N u-R a$ scaling, itself varies with $R a$ (e.g., Castaing et al. 1989; Grossman \& Lohse 2000; Kadanoff 2001). Another important issue is how the form of the probability distribution function for temperature fluctuations (PDF) scales with $R a$ and $P r$. Is it exponential or Gaussian?

Below about $R a \sim 10^{8}$, it is thought that flow in a Rayleigh-Benard cell has not become completely chaotic, but above $10^{8}$ the regime of so called "hard turbulence" is encountered. Characteristics of hard turbulence include (Xia \& Qiu 1997): 1) A Nusselt number $(N u)$ that scales as $R a^{2 / 7}$ (Castaing et al. 1989) instead of the classically expected $\left.R a^{1 / 3} ; 2\right)$ a PDF that is exponential, not Gaussian as might have been expected based on the central limit theorem (Kolmogorov 1962); and 3) coherent large-scale circulation. Experiments by Niemela et al. (2000) confirm these properties of hard turbulence across a large range of Rayleigh numbers, $10^{7}-10^{17}$. Numerical calculations by Rogers, Glatzmaier, \& Woosley (2003) confirm that at least the $R a-N u$ scaling characteristics of hard turbulence persist when the flow is compressible with density stratification.

However, our white dwarf problem is characterized by $R a$ still $10^{8}$ larger than studied by Niemela et al. More importantly perhaps, we are interested in convection without hard boundaries. Energy is dissipated in the star by expansion and neutrinos, both volumetric losses, not conductivity to a cold boundary. Heating also occurs over an extended region making for a very thick "wall zone" and a range of temperature fluctuations that is not limited by the temperature of some hot plate. In the Ia, the flow can also pass right through the core of the burning region and out the other side, allowing more complete mixing between the core and the convective region than is possible in RayleighBenard.

A hint of what may lie ahead at very high val- ues of Rayleigh number, comes from studies by Kraichnan (1962), and is referred to in the literature as the "ultimate" or "Kraichnan" regime of convection (Grossman \& Lohse 2000; Kadanoff 2001) . In situations where the effects of walls are suppressed, this transition may occur at much more modest values of $R a$, even $10^{6}$ (Lohse \& Toschi 2003). In this regime, it is thought that $N u \propto(\operatorname{RaPr})^{1 / 2}$ and that the large scale "rolls" seen in Rayleigh-Benard convection at lower $R a$ may give way to a more fragmented, chaotic pattern. It is unknown whether the distribution of temperature fluctuations in this regime is Gaussian or exponential.

It is noteworthy, however, that the $N u-R a$ scaling in this ultimate regime recovers the same simple scaling for $\Delta T$ found from mixing length, i.e., Eq.(23). That is, Eqs.(29), (30), and (33) plus the condition

$$
N u \sim R a^{1 / 2} \operatorname{Pr}^{1 / 2}
$$

implies that

$$
\Delta T=\left(\frac{\phi^{2} T}{\rho^{2} c_{\mathrm{P}}^{2} g l \delta_{\mathrm{P}}}\right)^{1 / 3},
$$

which to a factor of order unity is Eq.(23). This correspondence does not exist for any other scalings between $N u$ and $R a$, which in general would leave some residual dependence of $\Delta T$ on the conductivity or viscosity. The mixing length approximation is, apparently, equivalent to Kraichnan scaling. Might the other properties of near infinite $R$ a number circulation in Rayleigh-Benard cells also be relevant?

\subsection{Flow Patterns}

To a large degree, the velocities, temperature fluctuations, and, ultimately, the ignition process depend upon the circulating pattern assumed by the major flows within the core. We consider two representative cases - the "isotropic model" and the "dipole model". The actual solution may have aspects of both.

In the isotropic model, there is no preferred direction. Matter enters the burning region from all angles, is heated, reverses its direction and flows out. In the perfectly symmetric model, matter at the center is at rest, but this ideal state is 
never realized. Small imbalances in flow will result in overshooting, first in one direction, then another, so that the velocity vector at the origin varies in random way, being zero only on the average. A slice at constant radius of the convective flow might resemble the solar photosphere, except that the large entropy contrast between upward moving plumes and downflows would be absent. For high $R a$, the characteristic radial speed near the center would be less than, but perhaps not much less than that say a hundred $\mathrm{km}$ out. In this sense, the flow pattern might resemble the radial equivalent of Rayleigh-Benard in the "ultimate" or Kraichnan regime. Though not yet studied in the laboratory because of its inaccessibly high $R a$, it is hypothesized that in this regime plumes would travel from one plate to another in near ballistic fashion with the large scale circulation suppressed (Kadanoff 2001).

One might expect that in the absence of rotation there can be no preferred orientation, that is, this isotropic model would be the only physical one. However, numerical experiments (Kuhlen, Woosley, \& Glatzmaier 2003b; Paper 2) show that the convective flow in either a sphere or thick shell often takes on a dipole character. Matter flows in from one side, is heated in the central region, and flows out the other, like a jet engine. The center, far from being a point of stagnation, is characterized by the same high velocities found farther out in the convection zone. This is the stellar analogue of the large scale circulation, or "rolls", seen in Rayleigh-Benard convection. Similar dipole flows have been seen in three-dimensional studies of red giant convection by Woodward and colleagues (Porter, Anderson, \& Woodward 1997; Porter, Woodward, \& Jacobs 2000; Woodward, Porter, \& Jacobs 2002, 2003).

This dipole circulation is an example of spontaneous symmetry breaking. In the absence of rotation, the dipole picks an arbitrary axis, determined ultimately by tiny perturbations in the initial model, and maintains it for many convective turnover times. Over very long periods, the orientation of the dipole may vary due to the occasional large "intermittent" occurrence. Also, the average over many calculations with random starting conditions would give no preferred angle, so, in a sense, the model is still isotropic. But during the time the runaway develops, the main flow may be highly directional and this has important implications for the explosion that follows.

\section{IGNITION}

The runaway first commences when the temperature of the hottest fluctuation, $T_{\max }$, leads to nuclear heating faster than the adiabatic cooling that occurs when a blob crosses the burning region. Ignition will thus occur when the integral along a convective path,

$$
\int\left[\left(\frac{d T}{d r}\right)_{\exp }+\frac{\dot{S}_{\mathrm{nuc}}}{c_{\mathrm{P}} v_{\mathrm{rms}}}\right] d r
$$

diverges. From Eq. (14)

$$
\left(\frac{d T}{d r}\right)_{\exp } \approx-0.037 T_{c}\left(\frac{\rho_{9}}{2}\right)^{2 / 3} r_{7} .
$$

It remains to specify the velocity and some distribution of starting temperatures at the center of the star. As we shall see shortly ( $\S 4.3$ ), the average central temperature at ignition is in the range $T_{o, 8}=7.5-8.0$. From Eq. $(19), v_{\mathrm{rms}} \approx 60-100$ $\mathrm{km} \mathrm{s}^{-1}$. Taking $80 \mathrm{~km} \mathrm{~s}^{-1}$ as representative, we find that the first runaway will occur at a radius of over $300 \mathrm{~km}$ for a fluctuation hat started with a central temperature $T_{\max , 8}=8.623 \ldots$

This estimate is a little large. The initial perturbations in the star's center cannot have their temperature specified to arbitrary accuracy. As we shall see in $\S 4.3$, the typical variation in the temperature of the hottest few points in the core is a fraction of $\Delta T$, Eq.(23), about $1 \%$. Thus instead of $T_{\max , 8}=8.623$ one should realistically consider temperatures in the range $T_{\max , 8}=8.5-8.7$. This implies typical ignition radii in the range 150 - 200 $\mathrm{km}$, though larger values are still possible in rare cases.

The hottest fluctuations probably begin with (though do not necessarily end up with) radial velocities lower than the average. Longer residency in the burning region leads to higher temperature fluctuations. Doing the same calculation for an assumed average radial speed of $20 \mathrm{~km} \mathrm{~s}^{-1}$, one obtains ignition for hot fluctuations in the range $T_{\max , 8}=8.0-8.2$ at radii again near $200 \mathrm{~km}$. It is important for the issue of multi-point ignition that the igniting fluctuation takes from one to several seconds to reach its ignition radius (§ 4.4). 
This maximum fluctuation temperature at runaway, $T_{\max }$, is to be distinguished from the mean central temperature, $T_{o}$, in that it reflects conditions in a small, atypical fluid element out on the tail of the probability distribution function (PDF). Just how far out, is a critical issue.

\subsection{The probability density function for fluctuations}

In order to address this question, one needs to assess the probability that a given high temperature fluctuation will occur. Ignition does not happen when the average core temperature reaches a flash point, but when the hottest persistent fluctuation does.

We assume a simple model that should capture the essence of the real runaway. Divide the star into two regions: the burning core with mass $M_{\text {core }}$, and a larger reservoir, $M_{\text {conv }}$, of cooler matter to which it is convectively coupled. Because of the finite speed of convection, $M_{\text {conv }}$ shrinks during the final stages of the runaway while $M_{\text {core }}$ remains nearly constant, but we examine conditions during the last few seconds before the explosion when this ratio is approximately constant.

Matter passing through $\mathrm{M}_{\text {core }}$ gets a temperature increment, $\Delta T$, due to nuclear burning. The magnitude of this increment is sufficient, when mixed with $M_{\text {conv }}$, to heat the entire region by an amount $\Delta T$ during a convective mixing time. That is, each time the convection zone circulates once, one must generate an amount of energy equal to its adiabatic excess, $\Delta \nabla T l$, which is lost. Since $M_{\text {conv }} \gg M_{\text {core }}$, the time spent by a fluid element in the energy generating core, is much less than the convective mixing time, by approximately the ratio $M_{\text {core }} / M_{\text {conv }}$.

An operational value for this ratio comes from comparing the time it takes matter at the center of the star to appreciably increase its temperature in the presence or absence of convective cooling. From a one-dimensional calculation that employs a time-dependent mixing-length model for convection (the Kepler code, Weaver, Zimmerman, \& Woosley 1978), for a range of central temperatures $7.0 \lesssim T_{o, 8} \lesssim 7.5$ this ratio is approximately 50 . Very crudely this is also the the ratio of the mass within one pressure scale height, about 0.3 $\mathrm{M}_{\odot}$, to that inside $130 \mathrm{~km}$, the one-half energy generating region, about $0.01 \mathrm{M}_{\odot}$. Henceforth we adopt $M_{\text {conv }} / M_{\text {core }}=50$. The time that a fluid element resides in $M_{\text {core }}$ is approximately its radius divided by the convective speed, $\sim 100 \mathrm{~km} \mathrm{~s}^{-1}$, or 1 second. The total convective mixing time for $M_{\text {conv }}$ is thus $\sim 50$ seconds.

During each mixing event in $M_{\text {core, a certain }}$ fraction of the material, $f<1$, is exchanged. Given the efficient nature of convection and the turbulent nature of the core, $f$ may be close to unity. A representative value $0.9 \lesssim f \lesssim 0.99$ will be assumed. This implies that each mixing leaves behind between $1 \%$ and $10 \%$ of the mass in $M_{\text {core }}$. This residual matter increases its temperature beyond the average for the core, which in fact changes very little. After $n$ mixing events, each approximately $1 \mathrm{~s}$ in duration, the fraction of material that has increased its temperature by $n \Delta T$ is $(1-f)^{n}$. Such a power law naturally gives rise to an exponential PDF for temperature fluctuations in the core, such that the probability per unit volume (or mass) for finding a fluid element with temperature, $T$, when the average is $T_{o}$, is

$E P D F=-\frac{\ln (1-f)}{\Delta T} \exp \left[\ln (1-f)\left(T-T_{o}\right) / \Delta T\right]$

Here, the PDF has been normalized on the temperature domain $\left(T_{o}, \infty\right)$. This function gives the probability, per unit temperature, that a measurement at a random time and place will give a temperature, $T$. The integral of this function from $T>T_{o}$ to infinity is the fraction of the mass that has temperature $T$ or greater. Because of the simple toy model assumed here, the PDF is exponential (hence, "E" in EPDF), but one cannot exclude that it might instead be Gaussian. If the fluctuations are statistically independent of one another, the central limit theorem implies

$$
G P D F=\left(\frac{4}{\pi(F \Delta T)^{2}}\right)^{1 / 2} \exp \left(-\frac{\left(T-T_{o}\right)^{2}}{(F \Delta T)^{2}}\right)
$$

where $F<1$ is an uncertain factor, not much less than one, to be determined by experiment or simulation.

Unfortunately, the PDF by itself does not say how the temperature excess is distributed - in a few large blobs or very many small ones. For this, one needs additional information or assumptions. In particular, how many thermally discrete 
regions exist in the inner $100 \mathrm{~km}$ where the energy is mostly generated?

\subsection{Persistence of fluctuations}

A minimum size for the thermally discrete cell is capable of crossing the burning region while retaining its excess heat for a time, $\tau$, without cooling by conduction is $\lambda_{\text {cond }}$, given by

$$
\begin{aligned}
\tau & \approx \frac{\lambda_{\text {cond }}^{2}}{D}=\frac{\lambda_{\text {cond }}^{2} \rho c_{P}}{\sigma} \\
& \approx \frac{l}{v_{\mathrm{rms}}} \sim 1 \mathrm{~s},
\end{aligned}
$$

or $\lambda_{\text {cond }} \sim 10 \mathrm{~cm}$. Based upon this scale alone, the number of thermally discrete regions in $M_{\text {core }}$ would be very large, $\sim 10^{18}$, but turbulence changes this by making the size of the thermally discrete region much larger..

In a turbulent fluid, large eddies help to generate fluctuations which the small ones disrupt. Consequently, there is some cut off to the size spectrum of persistent fluctuations that depends on the intensity of turbulence in the medium. A relevant length scale is the distance over which the average temperature varies by a characteristic fluctuation scale. For a nearly adiabatic core,

$$
\lambda_{\text {turb }} \sim \frac{\Delta T}{\nabla T_{\mathrm{ad}}} .
$$

For $\Delta T$ given by Eq. (24) and $\nabla T_{\text {ad }}$ by Eq. (14), $\lambda_{\text {turb }}$ is about a $\mathrm{km}$.

Eddies smaller than this will tend to smooth out fluctuations larger than $\Delta T$, while larger ones will just move them around and create new ones. The number of such regions inside $100 \mathrm{~km}$ that might survive against turbulent mixing is about $10^{6}$. This is a more realistic estimate than the larger number given by conduction alone, and will be used in subsequent discussions. Fortunately the answer will only depend on the logarithm of this number.

Of course, the turbulence is not really homogeneous or isotropic. Porter \& Woodward (2000), in three-dimensional numerical studies of stellar convection up to $R a \sim 10^{12}$, find that the upwelling plumes, the ones that would carry the ignition sparks here, are considerably more laminar than the highly turbulent downdrafts. It is difficult to quantify this result for the present problem of white dwarf ignition, but regions of a $\mathrm{km}$ or so might survive turbulent dispersal for the several seconds it takes them to run away.

\subsection{Exponentiation of ignition points}

Once a single point has ignited, will more follow or will the runaway only ignite once? When the winner crosses the finish line, how close behind are the second, third, etc. runners?

We previously derived $(\S 4) T_{\max , 8} \approx 8.0-8.7$ as the central temperature of the unusually hot spark that finally ignites the runaway. We shall adopt $T_{\max , 8}=8.5$ in what follows, but similar results are obtained for other values in this range. At the time of interest, there will be a large number of points in the core with temperature close to, but less than $T_{8}=8.5$. In fact the average central temperature at that time will be $T_{o, 8}=8.5-n \Delta T$ where

$$
n=\frac{\log N}{|\log (1-f)|} .
$$

For $N=(100 \mathrm{~km} / \lambda)^{3} \sim 10^{6}$ and assuming an exponential PDF with $f=0.9-0.99, n=3-6$, which should be typical. Even if $N \sim 10^{18}$ and $f=0.9, n=18$, an extreme upper bound.

From Eq.(24) one can evaluate $\Delta T / T$ to determine the range of average central temperature when the runaway finally commences to be

$$
\begin{aligned}
T_{o, 8} & \approx 8.5-(3 \text { to } 6) \Delta T \\
& \approx 7.7-7.9
\end{aligned}
$$

The exponentiation time scale, that time during which the number of ignition points will rise one $e$-fold, is the time it takes for the temperature near $T_{8}=8.5-d T$ to rise by $\delta T$, where from Eq. (38),

$$
\delta T=\Delta T /|\ln (1-f)| .
$$

That is, for $f=0.9$ and an exponential PDF,

$$
\begin{aligned}
\tau_{\mathrm{exp}} & =\frac{c_{\mathrm{P}}}{S_{\mathrm{nuc}}} \delta T \\
& \approx 0.1 \mathrm{~s}\left(\frac{7.7}{T_{o, 8}}\right)^{23}\left(\frac{2}{\rho_{o, 9}}\right)^{3.3}\left(\frac{\delta T_{8}}{0.02}\right) .
\end{aligned}
$$

A PDF that is Gaussian(Eq. 39), or a larger value of $f$ would give a shorter time scale.

For reasonable choices of $f$ and $N$, the answer is considerably less than the time it takes a spark 
to traverse the convection region, about $1 \mathrm{~s}$, and comparable to the time it takes for the supernova itself to expand and shut off the runaway ( $\S 4.4)$. Hence it seems that multi-point ignition is favored, though, within current uncertainties, not guaranteed. The number of ignitions per second will be

$$
\dot{n}(t)=\exp \left(t / \tau_{\exp }\right)
$$

As noted previously, this answer is relatively insensitive to the uncertain value of $N$ (Eq. 42). Numerical calculations to confirm this result will need sufficient resolution to follow the evolution of temperature fluctuations far out on the tail of the PDF, but are badly needed.

\subsection{The end of ignition}

Nuclear burning in the core continues to raise the temperature for a time, but once ignition has occurred at any point, the spread of the flame rapidly reduces the binding energy of the white dwarf. Regions not yet encroached by flame are cooled by this expansion, shutting off the ignition. From that point onwards, the calculation is purely one of flame propagation.

When does this occur? We carried out a simulation of a $1.38 \mathrm{M}_{\odot}$ white dwarf at the onset of carbon runaway $\left(T_{o, 8}=7.6 ; \rho_{o, 9}=2.5\right)$ using the Kepler stellar evolution code (Weaver, Zimmerman, \& Woosley 1978). At that time, the energy input from nuclear burning, i.e., the convective luminosity had reached $8 \times 10^{45} \mathrm{erg} \mathrm{s}^{-1}$, in good agreement with Eq.(15). We then switched off nuclear energy generation and took a tiny time step, allowing the model to expand adiabatically with the existing velocity structure. From this, the rate of adiabatic reduction in central density appropriate to this luminosity was determined numerically,

$$
\frac{d \ln \rho}{d t}=-2.2 \times 10^{-4} \mathrm{~s}^{-1} .
$$

From the equation of state, the adiabatic relations for $d \ln \rho$ and $d \ln T$ are

$$
\begin{aligned}
& \frac{d \ln P}{d \ln \rho}=\Gamma_{1} \approx \frac{4}{3} \\
& \frac{d \ln T}{d \ln P}=\frac{\Gamma_{2}-1}{\Gamma_{2}} \approx 0.43 .
\end{aligned}
$$

So, expansion in the absence of nuclear burning reduces the temperature by

$$
\left(\frac{d \ln T}{d t}\right)_{\exp }=-1.2 \times 10^{-4} \mathrm{~s}^{-1} .
$$

Note that this relation between $\delta T$ and $\delta \rho$ is very different than Eq.(18) which is evaluated for nonadiabatic expansion at constant pressure.

This rate of cooling by expansion should be proportional to the change in net binding energy, and thus to total rate of energy deposition in the star. Once the flame forms, this occurs, off center, at a rapidly increasing rate. Because all motions are initially very subsonic, the whole star responds, even at its center, to energy deposited anywhere.

In the middle of the star, steady nuclear burning was actually raising the temperature at a rate that can also be determined numerically, with the energy generation turned back on,

$$
\left(\frac{d \ln T}{d t}\right)_{\mathrm{nuc}}=3.1 \times 10^{-2} \mathrm{~s}^{-1} .
$$

This implies that when the energy generation by the "flame sheet" becomes 250 times greater than $L=8 \times 10^{45} \mathrm{erg} \mathrm{s}^{-1}$, i.e., $2 \times 10^{48} \mathrm{erg} \mathrm{s}^{-1}$, expansion will start to quench the ignition.

A flame, once born, delivers energy at a rate

$$
\dot{\epsilon}=q_{\text {nuc }} A u \rho
$$

where $q_{\text {nuc }}$ is the energy released by carbon and oxygen fusing to iron, about $7 \times 10^{17} \mathrm{erg} \mathrm{g}^{-1}, A$ is its surface area, and $u$ is the average speed normal to the surface bounded by $A$. Niemeyer et al (1996), find, for single point off-center ignition, $u \sim 200 \mathrm{~km} \mathrm{~s}^{-1}$ after about $0.1 \mathrm{~s}$ when the burned region is about $10^{14} \mathrm{~cm}^{2}$ (their Fig. 4), so that $\dot{\epsilon} \sim 3 \times 10^{48}$ erg s$^{-1}$.

The ignition process thus ceases about $0.1 \mathrm{~s}$ after the first spark ignites off center. Other sparks, however, will still be in transit since it takes $\sim 1 \mathrm{~s}$ to emerge from the core $(\S 4)$. These hot, localized burning regions, already in the process of running away, will be more difficult to extinguish. We estimate that the total ignition process goes on for several tenths of a second.

\subsection{Angular distribution of the sparks}

Even though the exponentiation time scale in Eq.(45) is short and the number of potential ignition points large, there is some ambiguity in 
the counting of discrete points. If the dominant flow is, for example, dipole in nature ( $\S 3.2$ ), ignition will occur preferentially on one side of the star. The concept of multi-point ignition becomes blurred if those points are all in close proximity.

Indeed there may be great difficulty getting a viable supernova explosion if all the ignition occurs on one side (Niemeyer, Hillebrandt, \& Woosley 1996). Unless a transition to detonation occurs or pulsational oscillations, it will be difficult to ever burn the other side. The explosion will then be sub-energetic and produce too little ${ }^{56} \mathrm{Ni}$.

We speculate that the symmetric large scale flow, the dipole term in the case of spherical convection, is broken at the high Rayleigh number characteristic of the white dwarf as it runs away. In this sense the dipole flow in stellar convection is like the large cell-sized circulation seen in Rayleigh-Benard experiments. The flow is dominated by the largest symmetric scales. High Rayleigh number as in Eq. (29) may lead to diminished importance of both. It is also possible that rotation will diminish the effect of the dipole (Kuhlen, Woosley, \& Glatzmaier 2002).

If so, the fluctuations flowing out of the core may be not only numerous and small, but nearly isotropic. Experiments and numerical simulations to test this speculation are needed, but will be difficult.

\subsection{Further evolution of sparks}

Our calculations suggest multi-point ignition roughly $150-200 \mathrm{~km}$ off center. This is a small amount of mass, at most a few hundredths of a solar mass. Is the distinction between $r=0$ and $150 \mathrm{~km}$ important?

Yes, it is. Once a spark burns to iron, its density contrast, rather than being $\Delta \rho / \rho \sim 10^{-4}$ will be $15 \%$. The radial acceleration resulting from this buoyancy will be

$$
\begin{aligned}
g_{\mathrm{eff}} & \approx \frac{4 \pi G \rho r}{3}\left(\frac{\Delta \rho}{\rho}\right) \\
& \approx 8 \times 10^{8} \mathrm{r}_{7} \mathrm{~cm} \mathrm{~s}^{-2} .
\end{aligned}
$$

A blob starting off center accelerates rapidly and does most of its burning far out in the supernova. In a tenth of a second it is already moving several hundred $\mathrm{km} \mathrm{s}^{-1}$. One started dead center only expands initially at the laminar speed (or at a typical convective speed, which is comparable) and, in the same $0.1 \mathrm{~s}$, goes only about $10 \mathrm{~km}$.

\section{CONCLUSIONS}

Based upon a simple analytic description of the runaway, we expect that the first ignition will occur roughly $150-200 \mathrm{~km}$ off center when the hottest point in the center of the star reaches $8.0-8.7 \times 10^{8} \mathrm{~K}(\S 4)$. The average central temperature of the star when these fluctuations are produced is $T_{o, 8} \approx 7.7-7.9(\S 4.3)$. The orientation of this point with respect to the star's center may be given by the existence of a strong dipole asymmetry to the macroscopic convective flow just prior to the runaway $(\S 3.2)$.

The existence of this dipole component (§ 3.2), seen previously in numerical models of red-giant convection, in main sequence massive stars, and further explored in Paper II, is important in determining the outcome of the explosion. Even given multiple ignition points, if they all happen on the same side of the star in close proximity, and if there is no subsequent detonation, a weak explosion will ensue. It may be, however, that quasisymmetric flow is restored at higher values of $R a$ than have been simulated thus far. By analogy to Rayleigh-Benard convection, such a restoration of symmetry might be expected in the Kraichnan regime ( $\S 3.2)$. Alternatively, rotation may be invoked to alter the dipole flow (Paper II).

The convective speed, which gives a measure of the turbulent energy input on large length scales, is 50 to $100 \mathrm{~km} \mathrm{~s}^{-1}$ throughout most of the mass of the white dwarf when it explodes. This, not the laminar flame speed gives a minimum rate at which the flame spreads, even in the absence of strong Rayleigh-Taylor instability.

To carry the stellar luminosity at this speed, a superadiabatic excess, $\Delta T$, is required across the burning region. Mixing-length theory and Nusselt-Rayleigh scaling give a common prediction for the characteristic super-adiabatic excess, $\Delta T$, in the convection zone, i.e., $R a=(N u P r)^{1 / 2}$, only in the Kraichnan regime. All other scalings give a value for $\Delta T$ that depends upon the conductivity or viscosity or both. Assuming the validity of $(N u P r)^{1 / 2}$ scaling, and based upon a simple toy model ( $\S 4.1$ ), we show that the distribution of temperature fluctuations in the burning core may 
be exponential with a characteristic temperature scale $\lesssim \Delta T$. The hottest fluctuations are not produced by compression as in Höflich \& Stein (2002), but by extended residency and burning near the center of the star. We give an expression for the expected PDF, Eq.(38).

Once the first point has ignited, the delay until the second, third, etc. ignites is given by the time it takes a fluid element on the tail of the PDF to raise its temperature by an amount $\Delta T /|\ln (1-f)|$ where $f$ is the fraction of the core's mass that gets exchanged during a mixing time scale. For reasonable assumptions, this delay is $\tau_{\text {ign }} \sim 0.1 \mathrm{~s}$, Eq.(45). This is small compared with the time it takes the first igniting spark to transverse the burning region $(\sim 1 \mathrm{~s} ; \S 4)$ and comparable to the time for the expansion of the supernova to shut off the ignition process $(\S 4.4)$. The number of ignition events increases as approximately $\exp \left(t / \tau_{\text {ign }}\right)$. Thus multi-point ignition is possible.

However, an important unresolved issue is the angular distribution and mean separation of ignition points. This will be influenced by the dipole flow, but also by the propensity of hot regions to cluster.

Given that the number of ignitions and their location is exponentially sensitive, by Eq.(45), to small variations in the conditions in the core at the time of runaway, one expects that SN Ia models, starting from nearly identical circumstances will display chaotic variation in their properties, including their light curves. Factors that might cause a systematic variation in this dispersion include the carbon-mass fraction (Eqs.1 and 25) and the central density at ignition. Calculations to quantify these dependences will be difficult, but should some day be undertaken given the importance of Type Ia supernovae as standard candles for cosmology.

Our analytic study can also serve to motivate and guide future numerical work. First, because the issue of the dipole nature of the flow is important, all calculations should carry the entire $4 \pi$ solid angle of a sphere. Calculations that study just a wedge and assume spherical symmetry will miss an important aspect of the problem. Because of concerns that the dipole might be artificially created by the tendency of turbulence to cascade to large scales in $2 \mathrm{D}$ simulations, some calculations, at least, will need to be done in 3D.
A major goal of numerical simulation should be to ascertain the PDF for the temperature fluctuations. Is it exponential, as assumed here, or Gaussian? Is the characteristic scale given by $\Delta T$ in Eq.(44)? What is the operational value of $f$ ? What is the angular distribution and size distribution of the fluctuations? The Rayleigh number of such studies needs to be as high as possible, first because $R a$ in the star is naturally unapproachably high, and second, to see the dependencies of all the above answers, including the dipole flow, on $R a$. In Paper II we shall address some of these questions.

This work has been supported by the NSF (AST 02-06111), NASA (NAG5-12036), and the DOE Program for Scientific Discovery through Advanced Computing (SciDAC) under grant DEFC02-01ER41176. We greatly appreciate interesting discussions with Alan Kerstein, Jens Niemeyer, and Alex Heger that helped to clarify some of the ideas presented here.

\section{REFERENCES}

Alastuey, A., \& Jancovici, B. 1978. ApJ, 226, 1034

Arnett, W. D. 1969, Ap\&SS, 5, 280

Castaing, B., Guanaratne, G., Heslot, F., Kadanoff, L. et al. 1989, J. Fluid Mech, 204, 1

Caughlan, G. R., \& Fowler, W. A. 1988, ADNDT, 40, 283

Chiu, H.-Y. 1968, Stellar Physics, Ginn and Blaisdell publishing, Waltham, Mass., p. 120

Clayton, D. D. 1983, Principles of Stellar Evolution and Nucleosynthesis, (U. Chicago Press), p. 252.

Garcia-Senz, D., \& Woosley, S. E. 1995, ApJ, 454, 895

Glimm, J., \& Li, X. L. 1988, Phys. Fluids, 31, 2077

Grossman, S., \& Lohse, D. 2000, J. Fluid Mech., 407, 27.

Höflich, P., \& Stein, J. 2002, ApJ, 568779

Hoyle, F., \& Fowler, W. A. 1960, ApJ, 132, 565 
Hillebrandt, W., \& Niemeyer J. 2000, ARAA, 38, $191 b$

Höflich, P., \& Stein, J. 2002, ApJ, 568, 779

Kadanoff, L. P. 2001, Physics Today, 54(8), 34

Khokhlov, A., Oran, E. S., \& Wheeler, J. C. 1997, ApJ, 478, 678

Kolmogorov, A. N. 1962, J. Fluid Mech, 13, 82

Kraichnan, R. H. 1962, Phys. of Fluids, 5, 1374

Kuhlen, M., Woosley, S. E., \& Glatzmaier, G. 2003a, in preparation for ApJ

Kuhlen, M., Woosley, S. E., \& Glatzmaier, G. 2003b, in 3D-Stellar Evolution, eds. S. Turcotte, S. Keller, \& R. Cavallo, ASP Conf. Proc., 293, 147 (also astro-ph 0210557)

Lantz, S. R., \& Fan, Y. 1999, ApJ, 121, 247.

Nandkumar, R., \& Pethick, C. J. 1984, MNRAS, 209,511

Niemela, J. J., Skrbek, L., Sreenivasan, K. R., \& Donnelly, R. J. 2000, Nature, 404, 837

Niemeyer, J. C., Hillebrandt, W., \& Woosley, S. E. 1996, ApJ, 471, 903

Niemeyer, J. C., \& Woosley, S. E. 1997, ApJ, 475, 740

Niemeyer, J. C. 1999, ApJL, 523, L57

Nomoto, K., Sugimoto, D., \& Neo, S. 1976, Ap\&SS, 39L, 37

Nomoto, K., Thielemann, F.-K., \& Yokoi, K. 1984, ApJ, 286, 644

Porter, D. H., \& Woodward, P. R. 2000, ApJS, 127,159

Porter, D. H., Anderson, S. E., and Woodward, P. R., 1997, Access magazine, available only on the web, at http://www.lcse.umn.edu/research/RedGiant

Porter, D. H., Woodward, P. R., \& Jacobs, M. L., 2000, in Convection in Slab and Spheroidal Geometries, Proc. 14th International Florida Workshop in Nonlinear Astronomy and Physics: Astrophysical Turbulence and Convection, Ann. N. Y. Acad. Sci., 898, 1 (see also www.lcse.umn.edu/convsph)
Reinecke, M., Hillebrandt, W., \& Niemeyer, J. 1999, A\&A, 347, 739

Reinecke, M., Hillebrandt, W., \& Niemeyer, J. 2002a, New Astron. Rev., 46, 481

Reinecke, M., Hillebrandt, W., \& Niemeyer, J. 2002b, A\&A, 391, 1167

Rogers, T., Glatzmaier, G., \& Woosley, S. E. 2003, Phys. Rev. E, 67, in press

Timmes, F. X. 2000, ApJ, 528, 913

Timmes, F. X., \& Woosley, S. E. 1992, ApJ, 396, 649

Weaver, T. A., Woosley, S. E., \& Zimmerman, G. B. 1978, ApJ, 225, 1021

Woodward, P. R., Porter, D. H., \& Jacobs, M., 2002, in Proc. 3-D Stellar Evolution Workshop, UC Davis IGPP, in press, see also www.lcse.umn.edu/3Dstars.

Woodward, P. R., Porter, D. H., \& Jacobs, M 2003, in 3D Stellar Evolution, eds. S. Turcotte, S. Keller, \& R. Cavallo, ASP Conf Proc. 293, 45

Woosley, S. E. 1986, in Nucleosynthesis and Chemical Evolution, 16th Advanced Course, Saas Fee, ed. J. Audouze, C. Chiosi, \& A. Maeder, Swiss Soc Ap. and Astron.

Woosley, S. E. 1990, in Supernovae, ed. A. G. Petschek, (Berlin: Springer), 182

Woosley, S. E., \& Weaver, T. A. 1986, ARAA, 24, 205

Woosley, S. E. 2001, Nuclei in the Cosmos, 2000, Nuc. Phys. A 688, 9c

Woosley, S. E., Kuhlen, M., \& Glatzmaier, G. 2003, ApJ, in preparation, (Paper II).

Xia, K.-Q., \& Qiu, X.-L. 1997, PRL, 79, 5006

This 2-column preprint was prepared with the AAS LATEX macros v5.0. 